\title{
MONITORING ENVIRONMENTAL CHANGE IN AN ECOSYSTEM
}

\author{
M. Gámez ${ }^{\mathrm{a}}$, I. López ${ }^{\mathrm{a}}$, S. Molnár ${ }^{\mathrm{b}}$ \\ ${ }^{a}$ Departamento de Estadística y Matemática Aplicada, Universidad de Almería, \\ La Cañada de San Urbano,04120 Almería, España. ( mgamez@ual.es, \\ milopez@ual.es) \\ ${ }^{b}$ Institute of Mathematics and Informatics, Szent István \\ University, Páter K. u. 1., H-2103 Godollo, Hungary. (Molnar.Sandor@gek.szie.hu)
}

\begin{abstract}
The monitoring and analysis of the processes taking place in an ecosystem is a key issue for a sustainable human activity. A system of populations, as the biotic component of a complex ecosystem is usually affected by the variation of its abiotic environment. Even in nearly natural ecosystems an abiotic effect like climatic implications of global warming may cause important changes in the dynamics of the population system. In ecosystems involving field cultivation or any industrial activity; the abiotic parameter in question may be the concentration of a substance, changing e.g. as a result of pollution, application of a pesticide, or a fertilizer, etc. In many cases the observation of the densities of each population may be technically complicated or expensive, therefore the question arises whether from the observation of the densities of certain (indicator) populations, the whole state process of the population system can be uniquely recovered. The paper is aimed at a methodological development of the state monitoring, under the conditions of a changing environment. It is shown, how the technique of mathematical systems theory can be applied not only for the approximate calculation of the state process on the basis of the observed data, even under the effect of an exogene abiotic change with known dynamics; but in certain cases, also for the estimation of the unknown biological effect of the change of an abiotic parameter. The proposed methodology is applied to simple illustrative examples concerning a three-species predator-prey system.
\end{abstract}

Keywords: environmental monitoring, observer system, ecosystem

\section{INTRODUCTION}

Detection of the effect of human activities (e.g. pollution) and environmental change such as climatic variation, is an important part in the analysis of a composite system, including ecological, human and environmental subsystems. The effect of such factors on the parameters of population system models has been studied by several authors, see e.g. Gargani (2002), Xia (2007). The problem we consider is the following: Suppose we know that an abiotic change has an effect on the parameters of a population system, we want to estimate, at the same time both the whole state process and the change of the mentioned parameters, observing the densities of certain indicator populations. 
For the solution of this problem a constructive method is proposed. Mathematical Systems

Theory offers appropriate concepts and technique for the development such a methodology. The methodological foundations of the application of controllability and observability to frequency-dependent population models (described by systems with invariant manifold), have been set in Varga (1989) and Varga (1992), see also Scarelli and Varga (2002). The original problem of state monitoring of a population system as formulated in Varga et al. (2003) is that, from the observation of the time-dependent densities of certain species, the whole state process of the population system is to be recovered. An important concept for the solution of this problem is observability. The latter in this context means that from the observation of one or several (but not all) state variables, it is possible to recover the whole state process of the populations system, in a unique way (without determining, however, a constructive method to obtain this process.) Observability has been analysed in different population system models in Varga et al. (2002, 2003) and Shamandy (2005), see also López (2003), López et al. (2004). In order to prove observability, we use a general sufficient condition for local observability of nonlinear observation systems, published in Lee and Markus (1971). For a general review on the application of mathematical systems theory in population biology, see Varga (2008).

Once local observability near an existing equilibrium is proved, we also need a constructive method for the estimation of the state process. To this end an auxiliary system, the so-called observer system can be applied. The latter is constructed from the observed data, and asymptotically estimates the complete state process with exponential speed of convergence, see Sundarapandian (2002) and López et al. (2007a, b).

Now, if by human activities (e.g. pollution) any environmental disturbance takes place, the parameters of the population system model may change, which can be expressed in terms of unknown (constant or time-dependent) parameters, added to the known system parameters. In the paper we deal with cases. First, there is an unknown additive constant in certain biological parameters, and from the observation we estimate both these unknown constants, and the unknown solution of the population system model. In the second case, certain biological parameters change according to a known dynamics described by an additional system called exosystem. In Section 2, from nonlinear systems theory we briefly recall a concept and a theorem concerning observability. Section 3 is devoted to the observer design, and contains illustrative numerical examples for the application of the proposed methodology to both cases mentioned above. If we apply the above state process estimation to the composite system including both the population system model and the exosystem, we obtain asymptotic estimation not only for the state process of the population system, but at the same time also for the unknown parameters indicating the abiotic disturbances. Using computer simulation, the above construction will be illustrated by a threespecies Lotka-Volterra model, where the Malthus parameters are changed as result of a human (environmental) intervention. 


\section{CONCEPT OF OBSERVABILITY. APPLICATION TO A PREDATOR-PREY MODEL}

Given positive integers $m$, $n$, let

$$
f: \mathbf{R}^{n} \rightarrow \mathbf{R}^{n}, \quad h: \mathbf{R}^{n} \rightarrow \mathbf{R}^{m}
$$

be continuously differentiable functions and for some $x^{*} \in \mathbf{R}^{n}$ we have that $f\left(x^{*}\right)=0$ and $h\left(x^{*}\right)=0$.

We consider the following observation system

$$
\begin{aligned}
& \dot{x}=f(x) \\
& y=h(x),
\end{aligned}
$$

where $y$ is called the observed function.

Definition 2.1 Observation system (1)-(2) is called locally observable near equilibrium $x^{*}$, over a given time interval $[0, T]$, if there exists $\varepsilon>0$, such that for any two different solutions $x$ and $\bar{x}$ of system (1) with $\left|x(t)-x^{*}\right|<\varepsilon$ and $\left|\bar{x}(t)-x^{*}\right|<\varepsilon(t \in[0, T])$, the observed functions $h \circ x$ and $h \circ \bar{x}$ are different. ( $\circ$ denotes the composition of functions. For brevity, the reference to $[0, T]$ will be suppressed).

For the formulation of a sufficient condition for local observability consider the linearization of the observation system (1)-(2), consisting in the calculation of the Jacobians

$$
A:=f^{\prime}\left(x^{*}\right) \text { and } C:=h^{\prime}\left(x^{*}\right) \text {. }
$$

Theorem 2.2 (Lee and Markus, 1971). Suppose that

$$
\operatorname{rank}\left[C|C A| C A^{2}|\ldots| C A^{n-1}\right]^{T}=n .
$$

Then the observation system (1)-(1) is locally observable near the equilibrium $x^{*}$. We consider now, the biological model of 2 preys and 1 predator of the form $\dot{x}=f(x)$, determined by the following differential system

$$
\begin{aligned}
& \dot{x}_{1}=x_{1}\left(a_{1}-b_{11} x_{1}-b_{12} x_{2}\right) \\
& \dot{x}_{2}=x_{2}\left(-a_{2}+b_{21} x_{1}-b_{22} x_{2}+b_{23} x_{3}\right) \\
& \dot{x}_{3}=x_{3}\left(a_{3}-b_{32} x_{2}-b_{33} x_{3}\right)
\end{aligned}
$$

with $a_{i}, b_{i j}>0$ for all $i, j=1,2,3$. 
It is easy to provide a simple algebraic condition for the existence of an equilibrium in mathematical sense, however its positivity depends on the model parameters. Throughout the paper we shall suppose that there exists an equilibrium $x^{*}>0$ for the considered model.

We suppose that we observe the total quantity of population preys with distinction between them, i.e., the observation equation is

$$
\begin{gathered}
y=h(x):=\left(x_{1}-x_{1}{ }^{*}, x_{3}-x_{3}{ }^{*}\right) . \\
C=\frac{\partial h}{\partial x}\left(x^{*}\right)=\left(\begin{array}{lll}
1 & 0 & 0 \\
0 & 0 & 1
\end{array}\right)
\end{gathered}
$$

And linearizing the Lotka-Volterra system (4) we obtain

$$
A=\frac{\partial f}{\partial x}\left(x^{*}\right)=\left[\begin{array}{ccc}
-b_{11} x_{1}{ }^{*} & -b_{12} x_{1}{ }^{*} & 0 \\
b_{21} x_{1}{ }^{*} & -b_{22} x_{2}{ }^{*} & -b_{23} x_{2}{ }^{*} \\
0 & -b_{32} x_{3} & -b_{33} x^{*}
\end{array}\right]
$$

It is easy to check that, $\operatorname{rank}\left[C|C A| C A^{2}\right]^{T}=3$. Thus, by Theorem 2.2 the system is locally observable near the equilibrium, and the whole system state can be monitored observing only the total prey populations with distinction.

\section{CONSTRUCTION OF AN OBSERVER SYSTEM WITH ENVIRONMENTAL CHANGE}

Now, the construction of an observer system will be based on Sundarapandian (2002). Let us consider observation system (1)-(2).

Definition 3.1. Given a continuously differentiable function $G: \mathbf{R}^{n} \times \mathbf{R}^{m} \rightarrow \mathbf{R}^{n}$, system

$$
\dot{z}=G(z, y)
$$

is called a local asymptotic (respectively, exponential) observer for observation system (1)-(2) if the composite system (1)-(2),(5) satisfies the following two requirements.

i) If $x(0)=z(0)$, then $x(t)=z(t)$, for all $t \geq 0$.

ii) There exists a neighbourhood $V$ of the equilibrium $x^{*}$ of $\mathbf{R}^{n}$ such that for all $x(0), z(0) \in V$, the estimation error $z(t)-x(t)$ decays asymptotically (respectively, exponentially) to zero.

Theorem 3.2. (Sundarapandian, 2002). Suppose that the observation system (1)-(2) is Lyapunov stable at equilibrium, and that there exists a matrix $K$ such that matrix $A-K C$ is Hurwitz (i.e. its eigenvalues have negative real parts), where $A=f^{\prime}\left(x^{*}\right)$ and $C=h^{\prime}\left(x^{*}\right)$. Then dynamic system defined by

$$
\dot{z}=f(z)+K[y-h(z)]
$$


is a local exponential observer for observation system (1)-(2).

Now, for the estimation of a change in the dynamical parameters of an ecosystem, we recall that Sundarapandian (2002) also considered the possibility of an "input generator" determined by an external system called exosystem $w^{\prime}=s(w)$, in terms of which we can form a composite (nonlinear) system with inputs of the form

$$
\begin{aligned}
\dot{x} & =F(x, u(w)) \\
\dot{w} & =s(w) \\
y & =h(x),
\end{aligned}
$$

where we suppose that $F: \mathbf{R}^{n} \times \mathbf{R}^{k} \rightarrow \mathbf{R}^{n}, s: \mathbf{R}^{k} \rightarrow \mathbf{R}^{k}$ are continuously differentiable and $F\left(x^{*}, 0\right)=0, u\left(w^{*}\right)=0, s\left(w^{*}\right)=0$. Variable $u$ is interpreted as a time-dependent vector of system parameters of the original system (1), corresponding to right-hand side $f$. For the construction of an observer for the composite system we can apply the following

Theorem 3.3 (Sundarapandian, 2002). Suppose that observation system (7) is Lyapunov stable at equilibrium. If the system (7) has a local exponential observer, and that there exists a matrix $K$ such that matrix $A-K C$ is stable (its eigenvalues have negative real parts), where $A=F^{\prime}\left(x^{*}, w^{*}\right)$ and $C=h^{\prime}\left(x^{*}\right)$. Then dynamic system defined by

$$
\dot{z}=F(z, u(w))+K[y-h(z)]
$$

is a local exponential observer for observation system (7).

Applying this methodology, we can not only asymptotically recover the state process $x$ from the observation, but also estimate the unknown change of the model parameters of the population system.

To apply this construction to our population system (4), we remind that in López et al. (2007) it was proved that system (4) is asymptotically stable for an equilibrium $x^{*}=\left(x_{1}^{*}, x_{2}{ }^{*}, x_{3}^{*}\right)$. In fact, function

$$
V(x):=\alpha H\left(\frac{x_{1}}{x_{1}^{*}}\right)+\beta H\left(\frac{x_{2}}{x_{2}{ }^{*}}\right)+\gamma H\left(\frac{x_{3}}{x_{3}{ }^{*}}\right)-(\alpha+\beta+\gamma) \quad\left(x \in R_{+}^{3}\right),
$$

with

$$
\alpha=\frac{b_{21}}{x_{2}{ }^{*} x_{3}{ }^{*} b_{12}}, \beta=\frac{1}{x_{1}{ }^{*} x_{3}{ }^{*}}, \gamma=\frac{b_{23}}{x_{1}{ }^{*} x_{3}{ }^{*} b_{23}},
$$


and $H(v):=v-\ln v,\left(v \in \mathbf{R}_{+}\right)$was shown to be a Lyapunov function, implying asymptotic stability of equilibrium $x^{*}$ for system (4), globally with respect to the positive octant of $\mathbf{R}^{3}$.

\subsection{Observer for a system with unknown environmental change}

Assume that a considered ecosystem consists, on the one hand, of a system of several interacting populations living in the given habitat, and the abiotic environment on the other. The latter may also be exposed to climatic (e.g. seasonal) changes and/or human intervention, such as e.g. pollution, described by certain abiotic parameters (e.g. temperature or concentration). In this sections considering the predator-prey model (4), we suppose that the reference value of certain abiotic parameters change to an unknown constant value. The effect of this change will be described by a small additive term (disturbance) $w \in R$ in certain model parameters. In our illustrative numerical examples we will illustrate how we can recover the whole state process of the population system and estimate the unknown disturbance at the same time, by constructing and solving the corresponding observer system. In Example 1 we admit disturbance in all Malthus parameters, while in Example 1, the unknown disturbance appears in the interaction parameters.

Consider first our population system, completed with a trivial equation for $w$,

$$
\begin{aligned}
\dot{x}_{1} & =x_{1}\left(a_{1}+c_{1} w-b_{11} x_{1}-b_{12} x_{2}\right) \\
\dot{x}_{2} & =x_{2}\left(a_{2}+c_{2} w+b_{21} x_{1}-b_{22} x_{2}+b_{23} x_{3}\right) \\
\dot{x}_{3} & =x_{3}\left(a_{3}+c_{3} w-b_{32} x_{2}-b_{33} x_{3}\right) \\
\dot{w} & =0
\end{aligned}
$$

with $a_{i}, b_{i j}, c_{i}>0$ for all $i, j=1,2,3$.

It is clear that with the equilibrium $x^{*}>0$ of the previous section, $\left(x^{*}, 0\right)$ is an equilibrium for system (9).

Now, for the estimation of both components $(x, w)$ of system (9), i.e. both the state process and the unknown parameter, we suppose that the densities of both preys are observed:

$$
y=h(x)=\left(x_{1}-x_{1}^{*}, x_{3}-x_{3}^{*}\right) .
$$

Then

$$
C=\left(\begin{array}{llll}
1 & 0 & 0 & 0 \\
0 & 0 & 1 & 0
\end{array}\right), A=\left(\begin{array}{cccc}
-b_{11} x_{1}{ }^{*} & -b_{12} x_{1}{ }^{*} & 0 & c_{1} x_{1}{ }^{*} \\
b_{21} x_{2}{ }^{*} & -b_{22} x_{2}{ }^{*} & b_{23} x_{2}{ }^{*} & c_{2} x_{2}{ }^{*} \\
0 & -b_{32} x_{3} & -b_{33} x_{3}{ }^{*} & c_{3} x_{3}{ }^{*} \\
0 & 0 & 0 & 0
\end{array}\right) .
$$

Now, we easily obtain that $\operatorname{rank}\left[C|C A| C A^{2} \mid C A^{3}\right]^{T}=4$. Then by Theorem 2.2 , the system is locally observable near the equilibrium, and applying the theory of Sundarapandian (2002) we can construct a corresponding observer system. 
Example 1. As a numerical example, we consider the following two-prey onepredator model with the presence of an unknown environmental disturbance $w$ :

$$
\begin{aligned}
\dot{x}_{1} & =x_{1}\left(2+0.1 w-1.1 x_{1}-0.1 x_{2}\right) \\
\dot{x}_{2} & =x_{2}\left(-1-0.3 w+x_{1}-0.2 x_{2}+0.5 x_{3}\right) \\
\dot{x}_{3} & =x_{3}\left(3.6+w-0.8 x_{2}-0.7 x_{3}\right) \\
\dot{w} & =0 .
\end{aligned}
$$

System (11) has an equilibrium: $x^{*}=(1.4608,3.9307,0.6506,0)$, and with

$$
K:=\left(\begin{array}{cccc}
0 & 0 & 0 & 1 \\
0 & 0 & 0 & 0.1
\end{array}\right)^{T}
$$

matrix $A-K C$ is Hurwitz, therefore by Theorem 3.2 we can construct the following observer system

$$
\begin{aligned}
& \dot{z}_{1}=z_{1}\left(2+0.1 z_{4}-1.1 z_{1}-0.1 z_{2}\right) \\
& \dot{z}_{2}=z_{2}\left(-1-0.3 z_{4}+z_{1}-0.2 z_{2}+0.5 z_{3}\right) \\
& \dot{z}_{3}=z_{3}\left(3.6+z_{4}-0.8 z_{2}-0.7 z_{3}\right) \\
& \dot{z}_{4}=\left[y-\left(z_{1}-x_{1}{ }^{*}, z_{3}-x_{3}^{*}\right)\right](1,0.1) .
\end{aligned}
$$

If we suppose that environmental perturbation corresponds to the value $w=0.2$ and we take an initial condition $\left(x_{0}, w_{0}\right):=(1.3,3.1,0.4,0.2)$, near the equilibrium of system (11), and similarly, we consider another nearby initial condition, $z_{0}:=(1.8,3.5,0.7,0.4)$ for the observer system (12). Figure 1 shows that the corresponding solution $z$ tends to the solution $x$ of the original system.

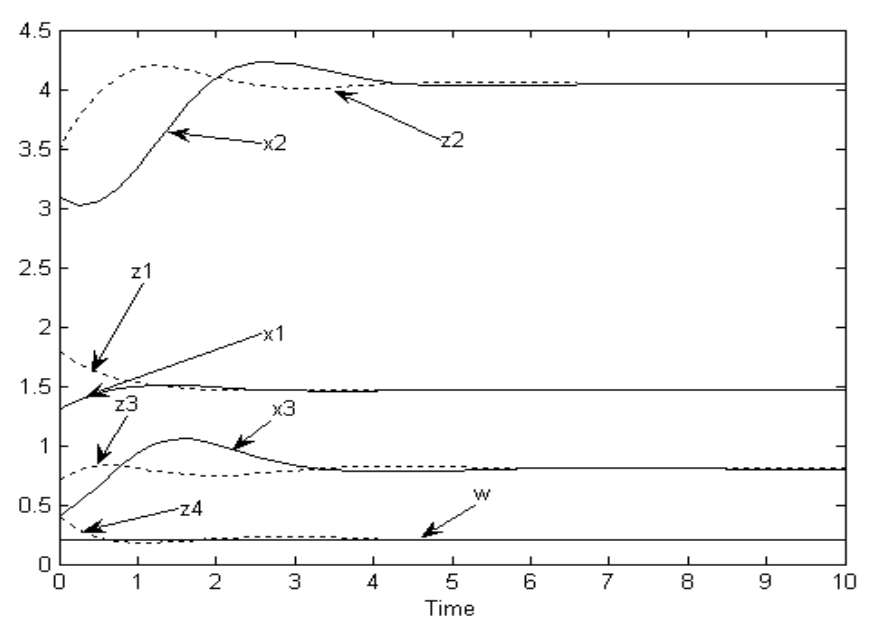

Figure 1. Solutions of systems (11) and (12) 
Let us now consider the case when there is an environmental disturbance in each interspecific interaction parameter. We then have a system of the following form:

$$
\begin{aligned}
& \dot{x}_{1}=x_{1}\left(a_{1}-b_{11} x_{1}-\left(b_{12}+c_{12} w\right) x_{2}\right) \\
& \dot{x}_{2}=x_{2}\left(a_{2}+\left(b_{21}+c_{21} w\right) x_{1}-b_{22} x_{2}+\left(b_{23}+c_{23} w\right) x_{3}\right) \\
& \dot{x}_{3}=x_{3}\left(a_{3}-\left(b_{32}+c_{32} w\right) x_{2}-b_{33} x_{3}\right) \\
& \dot{w}=0
\end{aligned}
$$

with $a_{i}, b_{i j}, c_{i j}>0$ for all $i, j=1,2,3$. As in the previous case, is clear that with the equilibrium $x^{*}>0$ of Section $2,\left(x^{*}, 0\right)$ is an equilibrium for system (13). Observing again the two prey populations, we have

$$
y=h(x)=\left(x_{1}-x_{1}^{*}, x_{3}-x_{3}^{*}\right) \text {. }
$$

Then with matrices

$$
\begin{aligned}
C & =\left(\begin{array}{llll}
1 & 0 & 0 & 0 \\
0 & 0 & 1 & 0
\end{array}\right), \\
A & =\left(\begin{array}{cccc}
-b_{11} x_{1}{ }^{*} & -\left(b_{12}+c_{12} w\right) x_{1}{ }^{*} & 0 & -c_{12} x_{1}{ }^{*} x_{2}{ }^{*} \\
\left(b_{21}+c_{21} w\right) x_{2}{ }^{*} & -b_{22} x_{2} & \left(b_{23}+c_{23} w\right) x_{2}{ }^{*} & c_{21} x_{1}{ }^{*} x_{2}{ }^{*}+c_{23} x_{2}{ }^{*} x_{3} \\
0 & -\left(b_{32}+c_{32} w\right) x_{3}{ }^{*} & -b_{33} x_{3}{ }^{*} & -c_{32} x_{2}{ }^{*} x_{3}{ }^{*} \\
0 & 0 & 0 & 0
\end{array}\right)
\end{aligned}
$$

we have again have $\operatorname{rank}\left[C|C A| C A^{2} \mid C A^{3}\right]^{T}=4$. Then by Theorem 2.2, we have observability, furthermore a corresponding observer system can be constructed, as illustrated by the following

Example 2. We consider the following two-prey one-predator model with the presence of an unknown environmental change effect $w$

$$
\begin{aligned}
\dot{x}_{1} & =x_{1}\left(2-1.1 x_{1}-(0.1+0.1 w) x_{2}\right) \\
\dot{x}_{2} & =x_{2}\left(-1+(-1+0.2 w) x_{1}-0.2 x_{2}+(0.5+0.3 w) x_{3}\right) \\
\dot{x}_{3} & =x_{3}\left(3.6-(0.8+0.3 w) x_{2}-0.7 x_{3}\right) \\
\dot{w} & =0
\end{aligned}
$$

System (15) has the same equilibrium: $x^{*}=(1.4608,3.9307,0.6506,0)$ as in Example 1 , and now with

$$
K:=\left(\begin{array}{cccc}
1 & 1 & 0 & 0 \\
0 & 0 & 1 & -1
\end{array}\right)^{T}
$$


matrix $A-K C$ is Hurwitz, providing the following observer system

$$
\begin{aligned}
\dot{z}_{1} & =z_{1}\left(2-1.1 x_{z}-(0.1+0.1 w) x_{2}\right)+\left[y-\left(z_{1}-x_{1}{ }^{*}, z_{3}-x_{3}{ }^{*}\right)\right](1,0) \\
\dot{z}_{2} & =z_{2}\left(-1+(1+0.2 w) z_{1}-0.2 z_{2}+(0.5+0.3 w) z_{3}\right)+\left[y-\left(z_{1}-x_{1}{ }^{*}, z_{3}-x_{3}{ }^{*}\right)\right](1,0) \\
\dot{z}_{3} & =z_{3}\left(3.6-(0.8+0.3 w) z_{2}-0.7 z_{3}\right)+\left[y-\left(z_{1}-x_{1}{ }^{*}, z_{3}-x_{3}{ }^{*}\right)\right](0,1) \\
\dot{w} & =0+\left[y-\left(z_{1}-x_{1}{ }^{*}, z_{3}-x_{3}{ }^{*}\right)\right](0,-1)
\end{aligned}
$$

Calculating with the initial values $\left(x_{0}, w_{0}\right)=(1.3,3.1,0.4,0.2) \quad$ and $z_{0}=(1.5,3,0.5,0.3)$ as in the previous example, we obtain the required approximations seen in Figure 2.

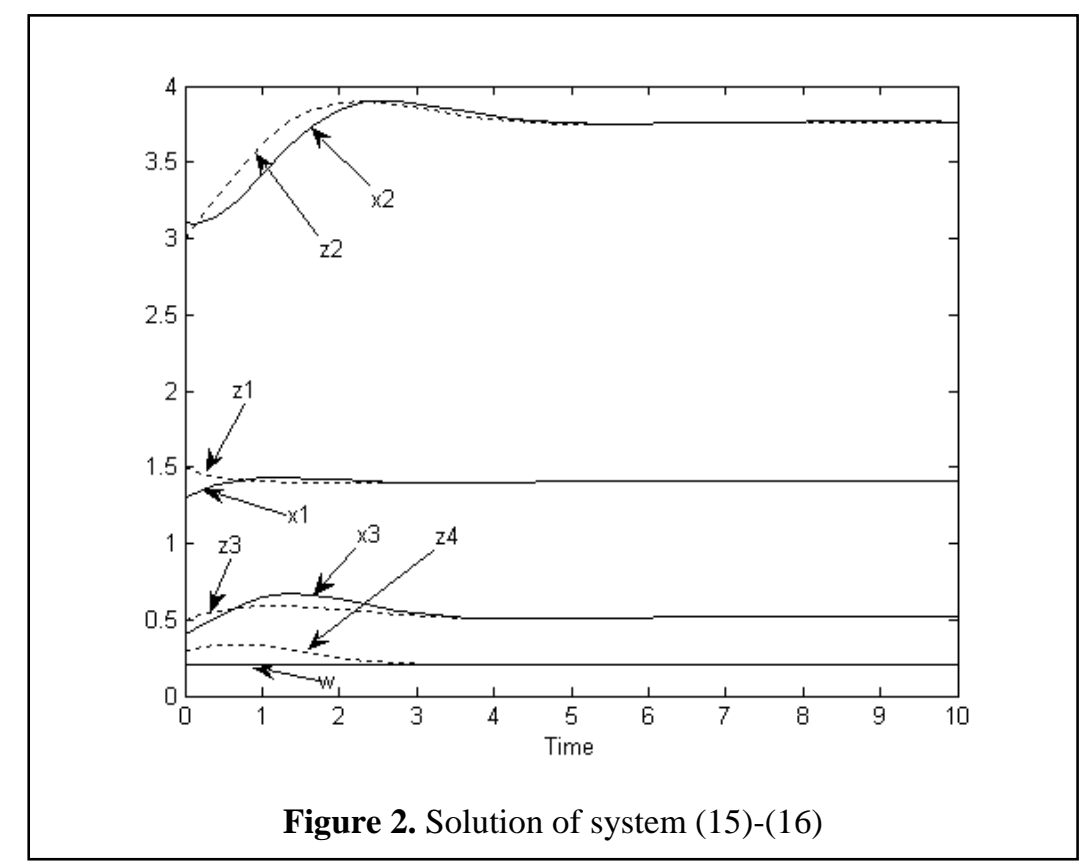

\subsection{Observer for a system with exosystem describing environmental change}

In this section we will design observer for a population system, supposing that in the abiotic environment there is a continuous change obeying a known dynamic law, which affect certain parameters of the population system. This abiotic process may be e.g. pollution produced by an industrial plant, a periodical (seasonal) change of temperature, or a monotonous increase of temperature due to global warming etc. In our illustrative examples below, this dynamics will be described by different differential equations. The situation can be handled in terms of a composite observation system of the form (7), with the above abiotic dynamics as exosystem.

First we assume that, with known positive constants $\alpha$ and $\delta$, the external system 


$$
\begin{aligned}
& \dot{w}_{1}=\alpha w_{2} \\
& \dot{w}_{2}=-\delta w_{1}
\end{aligned}
$$

describes a periodic change that affects the coefficients of the interaction between predator and preys in the following form

$$
\begin{aligned}
& \dot{x}_{1}=x_{1}\left(a_{1}-b_{11} x_{1}-\left(b_{12}+c_{12} w_{1}\right) x_{2}\right) \\
& \dot{x}_{2}=x_{2}\left(-a_{2}+\left(b_{21}+c_{21} w_{1}\right) x_{1}-b_{22} x_{2}+\left(b_{23}+c_{23} w_{1}\right) x_{3}\right) \\
& \dot{x}_{3}=x_{3}\left(a_{3}-\left(b_{32}+c_{32} w_{1}\right) x_{2}-b_{33} x_{3}\right) \\
& \dot{w}_{1}=\alpha w_{2} \\
& \dot{w}_{2}=-\delta w_{1},
\end{aligned}
$$

where all $c_{i j}$-s are positive. Since zero is a Lyapunov stable equilibrium of system (17), by a known result (see e.g. Isidori, 1995), equilibrium $\left(x^{*}, 0\right)$ system (18) is Lyapunov stable. With the same observation $y=h(x)=\left(x_{1}-x_{1}{ }^{*}, x_{3}-x_{3}{ }^{*}\right)$ as in the previous examples, and following Theorem 3.3, we can construct an observer for this system.

Example 3. We consider the system

$$
\begin{aligned}
& \dot{x}_{1}=x_{1}\left(2-1.1 x_{1}-\left(0.1+0.3 w_{1}\right) x_{2}\right) \\
& \dot{x}_{2}=x_{2}\left(-1+\left(1+0.2 w_{1}\right) x_{1}-0.2 x_{2}+\left(0.5+0.3 w_{1}\right) x_{3}\right) \\
& \dot{x}_{3}=x_{3}\left(3.6-\left(0.8+0.8 w_{1}\right) x_{2}-0.7 x_{3}\right) \\
& \dot{w}_{1}=1.3 w_{2} \\
& \dot{w}_{2}=-2.1 w_{1}
\end{aligned}
$$

Now using matrix

$$
K:=\left(\begin{array}{lll}
1 & 0 & 0 \\
0 & 0 & 0
\end{array}\right)^{T}
$$

we obtain the following observer:

$$
\begin{aligned}
& \dot{z}_{1}=z_{1}\left(2-1.1 z_{1}-\left(0.1+0.3 w_{1}\right) z_{2}\right)+\left[y-\left(z_{1}-x_{1}{ }^{*}, z_{3}-x_{3}^{*}\right)\right](1,0) \\
& \dot{z}_{2}=z_{2}\left(-1+\left(1+0.2 w_{1}\right) z_{1}-0.2 z_{2}+\left(0.5+0.3 w_{1}\right) z_{3}\right) \\
& \dot{z}_{3}=z_{3}\left(3.6-\left(0.8+0.8 w_{1}\right) z_{2}-0.7 z_{3}\right) \\
& \dot{w}_{1}=1.3 w_{2} \\
& \dot{w}_{2}=-2.1 w_{1}
\end{aligned}
$$

For the solution of the composite system (19), we set initial value $\left(x_{0}, w_{0}\right):=(1.3,3.1,0.4,0.1,0.1)$, and calculate the solution of the observer system (20) with initial value $z_{0}:=(1.8,3.5,0.7)$. Figure 3 shows how the state process is approximated by the solution of the observer even in case of variable environment. 


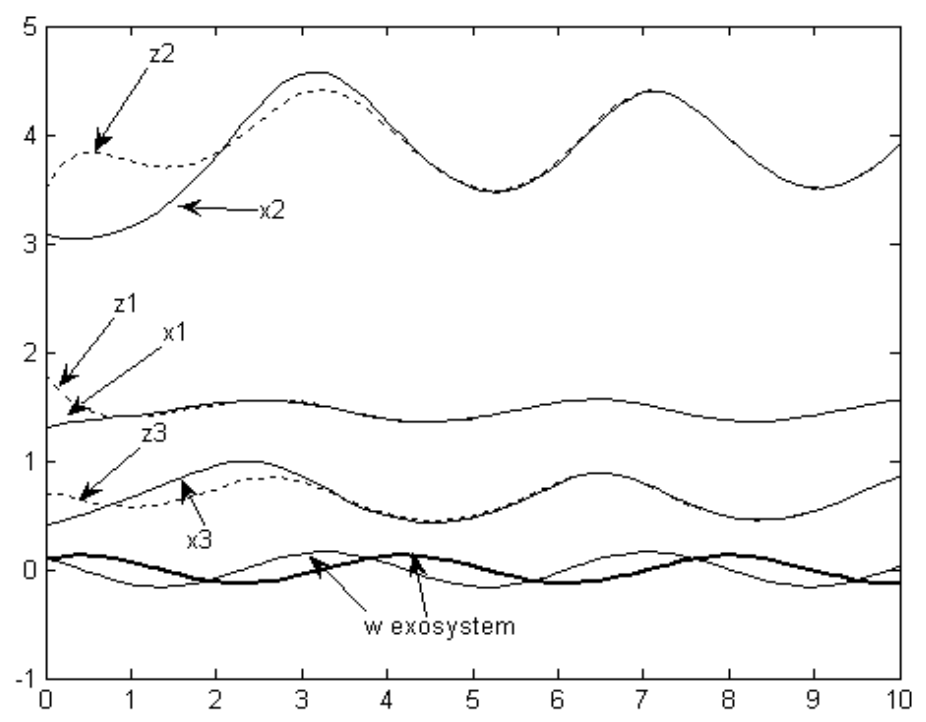

Figure 3. Solutions of systems (19) and (20)

In our last example we consider a case when an abiotic parameter, e.g. temperature displays a limited growth, described by a logistic equation. Assume that both the reproduction rate of the prey populations and the death rate of the predator increase with temperature. Such an abiotic effect can be described by the following composite system:

$$
\begin{aligned}
& \dot{x}_{1}=x_{1}\left(a_{1}+c_{1} w-b_{11} x_{1}-b_{12} x_{2}\right) \\
& \dot{x}_{2}=x_{2}\left(-a_{2}-c_{2} w+b_{21} x_{1}-b_{22} x_{2}+b_{23} x_{3}\right) \\
& \dot{x}_{3}=x_{3}\left(a_{3}+c_{3} w-b_{32} x_{2}-b_{33} x_{3}\right) \\
& \dot{w}=\alpha(1-\delta w),
\end{aligned}
$$

where $c_{i}>0$ for $i=1,2,3$.

Since $w^{*}:=1 / \delta$ is an asymptotically stable equilibrium for $\dot{w}=\alpha(1-\delta w)$, equilibrium $\left(x^{*}, w^{*}\right)$ is Lyapunov stable near its equilibrium for the composite system (20). Therefore, for the usual observation

$y=h(x)=\left(x_{1}-x_{1}^{*}, x_{3}-x_{3}^{*}\right)$,

we can construct an observer for a concrete system in

Example 4. Consider the system 


$$
\begin{aligned}
\dot{x}_{1} & =x_{1}\left(2+0.1 w-1.1 x_{1}-0.1 x_{2}\right) \\
\dot{x}_{2} & =x_{2}\left(-1-0.3 w+x_{1}-0.2 x_{2}+0.5 x_{3}\right) \\
\dot{x}_{3} & =x_{3}\left(3.6+0.6 w-0.8 x_{2}-0.7 x_{3}\right) \\
\dot{w} & =0.3(1-0.025 w)
\end{aligned}
$$

Now, with

$$
K=\left(\begin{array}{ccc}
0 & 0 & 0 \\
0 & 0 & 0.1
\end{array}\right)^{T}
$$

matrix $A-K C$ is Hurwitz, and the corresponding observer is

$$
\begin{aligned}
\dot{z}_{1} & =z_{1}\left(2+0.1 w-1.1 z_{1}-0.1 z_{2}\right) \\
\dot{z}_{2} & =z_{2}\left(-1-0.3 w+z_{1}-0.2 z_{2}+0.5 z_{3}\right) \\
\dot{z}_{3} & =z_{3}\left(3.6+0.6 w-0.8 z_{2}-0.7 z_{3}\right) \\
\dot{w} & =0.3(1-0.025 w)+\left[y-\left(z_{1}-x_{1}{ }^{*}, z_{3}-x_{3}{ }^{*}\right)\right](0,0.1)
\end{aligned}
$$

The initial condition is $\left(x_{0}, w_{0}\right):=(2,6,7,11)$ for system $(22)$ and $z_{0}:=(1,5,6)$ for its observer system (23). , we can check that the solution of the original system is recovered by the observer system, see Figure 4.

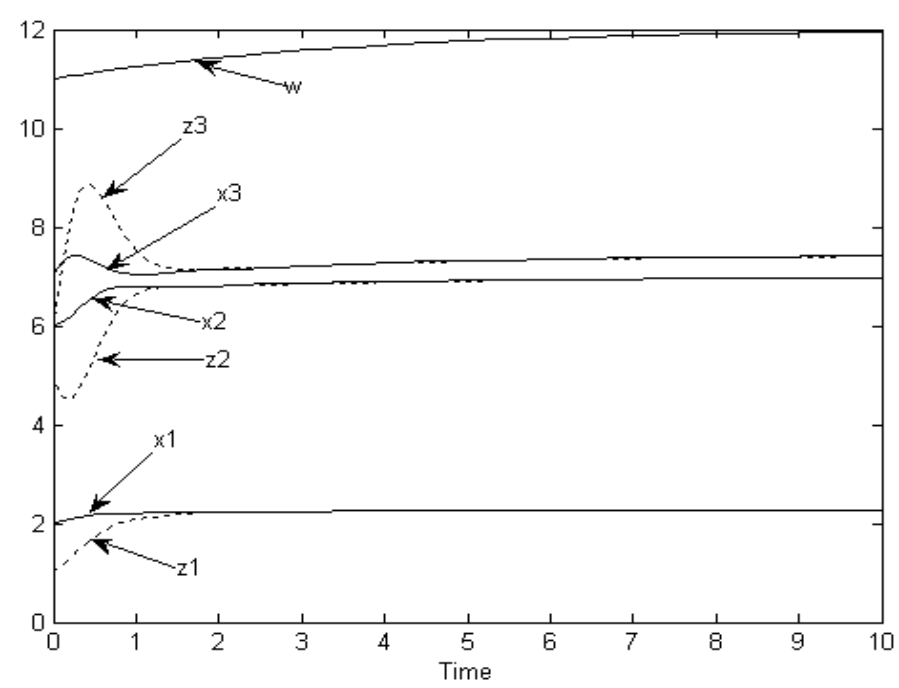

Figure 4. Solution of system (22) and (23)

\section{DISCUSSION}

As a continuation of our recent research line, the monitoring methodology has been further developed towards models with changing environment. With the applied methodology, monitoring is not restricted to the estimation of the population state process or the detection of a change in a constant (intrinsic growth rate or predation 
rate) of the population system model, but also makes possible to trace the biological consequences of a continuous change in certain abiotic environmental factors, including industrial pollution and climate change.

The problem of recovering an unknown solution of the population system model, is clearly equivalent to the problem of having information about its initial value at the beginning of the observation period. Therefore, the initial value for the observer is taken arbitrary but near the equilibrium.

We note that our approach can be naturally extended from the presented simple illustrative ecosystem model to more complex models even of non Lotka-Volterra type, e.g. to trophic chains with structure resource - producer - primary consumer, see Samandy (2005).

For the application to real field data, our approach may also need an extension to discrete-time ecosystem models, based on nonlinear discrete-time observer design, see e.g. Sundarapandian (2005).

\section{ACKNOWLEDGEMENTS}

The authors wish to thank the Ministry of Education and Science of Spain for the financial support of the project TIN2007-67418-C03-02, which has partially supported this work. The research was also supported by the Hungarian National Scientific Research Fund (OTKA K 68187).

\section{REFERENCES}

Isidori, A. Non linear control systems, Springer, New York, 1995.

López, I.. Observabilidad y controlabilidad en modelos de evolución (Tesis doctoral). Almería: Servicio de Publicaciones de la Universidad de Almería. ISBN: 84-8240683-3. 2003.

López, I., Gámez, M. and Carreño, R. Observability in dynamic evolutionary models. Biosystems, 73, 99-109. 2004.

López I, Gámez M, Molnár. Observability and observers in a food web. Applied Mathematics Letters 20 (8): 951-957. 2007a

López, I., Gámez, M., Garay, J. and Varga, Z. Monitoring in a Lotka-Volterra model. Biosystems, 83, 68-74. 2007b.

Lee, E.B. and Markus, L. Foundations of Optimal Control Theory. Wiley New York. 1971.

Scarelli, A., Varga, Z. Controllability of selection-mutation systems. BioSystems 65 (2002), No 2-3, 113-121.

Shamandy, A. Monitoring of trophic chains. Biosystems, Vol. 81, Issue 1, 43-48. 2005. Sundarapandian, V. Local observer design for nonlinear systems. Mathematical and computer modelling 35, 25-36. 2002. 
Sundarapandian, V. Nonlinear observer design for a general class of discrete-time nonlinear systems with real parameter disturbance. Computers and Mathematics with applications 49 (2005) 1177-1194.

Varga, Z., On controllability of Fisher's model of selection. In "Differential Equations" (Eds. C. M. Dafermos, G. Ladas, G. Papanicolau) Marcel Dekker, New York (1989) 717-723.

Varga, Z. On observability of Fisher's model of selection. Pure Math. and Appl. Ser. B, 1, 15-25. 1992.

Z. Varga, Applications of mathematical systems theory in population biology. Periodica Mathematica Hungarica , Vol. 56 (1), (2008) 157-168.

Varga, Z., Scarelli, A. and Shamandy, A. An observability problem of population ecology. In: V. Capasso (Ed.), Mathematical Modelling and Computing in Biology and Medicine, 5th ECMTB Conference 2002 (Abstracts, p.189). MIRIAM, Milan, 2002.

Varga, Z., Scarelli, A. and Shamandy, A. State monitoring of a population system in changing environment. Community Ecology 4 (1), 73-78. 2003. 